\title{
Labioplastía genital, experiencia y resultados en 500 casos consecutivos
}

\author{
Jack Pardo S. ${ }^{1}$, Vicente Solá $D .^{2}$, Guillermo Galán $C .^{3}$, Luis Contreras $M .^{4}$ \\ ${ }^{1}$ Ginecólogo de Clínica Sara Moncada y Clínica Lo Curro, ${ }^{2}$ Ginecólogo de Clínica Las Lilas y Lo Curro, ${ }^{3}$ Ginecólogo \\ Profesor Asistente de Obstetricia y Ginecología Universidad de Chile y Docente Universidad de Santiago de Chile, \\ ${ }^{4}$ Anatomopatólogo Laboratorio de Anatomía Patológica y Patología Molecular C y S.
}

\section{RESUMEN}

Antecedentes: La cosmetoginecología se ha instalado, no exenta de controversia, en la práctica ginecológica durante la última década. Objetivo: Presentar los resultados obtenidos en una serie de 500 labioplastías de labios menores realizadas por dos cirujanos en el curso de 10 años. Método: Estudio descriptivo del universo de labiobioplastías practicadas por dos cirujanos entre octubre de 2003 y abril de 2014. La decisión de intervención quirúrgica se basó en razones estéticas, funcionales y psicológicas, y no por una clasificación de hipertrofia de labios menores. Resultados: Aproximadamente la mitad se realizó utilizando láser y la otra mitad electrobisturí, sin ventajas de alguna técnica sobre la otra, en cuanto a complicaciones, grado de satisfacción y resultado final estético. Las indicaciones fueron: estética en 95,4\% de las intervenciones, funcional en $37,2 \%$ y psicológicas en $17,4 \%$. Los resultados fueron satisfactorios en el $99 \%$ de las pacientes, según un autoreporte. No hubo complicaciones importantes y sólo en dos casos se presentó hemorragia post operatoria que requirió una sutura. El procedimiento, cuando fue único, fue ambulatorio, requiriendo no más de 8 horas de hospitalización. En esta experiencia, el $46 \%$ se asoció a otra cirugía ginecológica. Conclusión: A nuestro parecer, la hipertrofia de labios menores, aún cuando pueda considerarse subjetiva, es una condición que merece ser corregida cuando es requerido por la paciente y que las técnicas actuales ofrecen resultados seguros y satisfactorios.

\section{PALABRAS CLAVE: Cirugía cosmética genital femenina, labioplastía, cosmetoginecología, hipertrofia de labios menores, labioplastía láser, rejuvenecimiento vulvar/vaginal}

\section{SUMMARY}

Background: Cosmetogynecology is installed, not without controversy, in gynecological practice in the last decade. Aims: We present a descriptive study of 500 labia minora labiaplasty performed by two surgeons in the course of 10 years. Method: A descriptive study of the universe of labiobioplastys performed by two surgeons between October 2003 and April 2014. The decision for surgery was based on aesthetic, functional and psychological reasons and not on a classification of labia minora hypertrophy. Results: Approximately, half was performed using laser, and electrocautery the other half, without any technical advantages over the other, in terms of complications, satisfaction and aesthetic final outcome. Indications for surgery were: in $95.4 \%$ of interventions, functional in $37.2 \%$ and $17.4 \%$ psychological. The results were satisfactory in $99 \%$ of patients, according to a self-report. There were no major complications and only two cases presented postoperative bleeding that required stitches. The procedure was ambulatory when it was unique, requiring no more than 8 hours of hospitalization. In this experience, $46 \%$ was associated with another gynecological surgery. Conclusion: In our view, the labia minora hypertrophy, even though it may be considered subjec- 
tive, is a condition that should be corrected as required by the patient and the current techniques provide safe and satisfactory results.

\section{KEY WORDS: Female genital cosmetic surgery, labiaplasty, cosmetogynecology, laser labiaplasty, labia minora hypertrophy, vaginal/vulvar rejuvenation}

\section{INTRODUCCIÓN}

Desde hace casi dos décadas se agregaron al conocimiento y práctica de la ginecología, una serie de procedimientos que se engloban dentro de lo que se ha denominado cosmetoginecología o cirugía cosmética genital femenina (1). Sin embargo, a la fecha existen pocos estudios y revisiones al respecto que permitan juzgar sus resultados (2-4).

En el caso de la labioplastía, o reducción quirúrgica de labios menores ( $y$, con frecuencia, de parte del prepucio del clítoris), ésta se realizaba en casos excepcionales, principalmente en niñas y adolescentes con hipertrofia de ninfas. La labioplastía está indicada en hipertrofia de labios menores; esto es, cuando miden más de $4 \mathrm{~cm}$ en extensión y ante molestias funcionales y/o estéticas (2). Sus causas no están claramente definidas, pero se asocian a factores tales como: uso de hormonas androgénicas, desbalances hormonales, irritación local crónica, condición congénita o idiopática que corresponde a la mayoría de los casos (5-7).

Las mujeres consultan generalmente motivadas por una condición estética, pero también funcional y psicológica. No existen mediciones de cada motivo en particular, pero con frecuencia se encuentran combinadas $(8,9)$. En el caso de condiciones psicológicas, es habitual encontrar mujeres que rechazan ciertas prácticas sexuales (como el cunnilingus), debido al aspecto de sus genitales externos (1). En nuestra experiencia, no es infrecuente que mujeres sobre 25 años confiesen que su virginidad se relaciona a un problema de autoestima por el tamaño de sus labios menores.

Existen muchas técnicas utilizadas para realizar la labioplastía, las que varían desde el método para cortar hasta el diseño de las líneas de corte. Se han comunicado labioplastías realizadas con bisturí, tijera, láser y electrobisturí. Así también, las líneas de corte varían desde las labioplastías en "Z", resecciones laterales o en triángulo. No hay comunicaciones randomizadas que permitan evidenciar ventajas de un instrumento y/o líneas de corte y éstas varían según el autor.

En 2003 iniciamos una experiencia en labioplastía con láser, reportada en 2005 con 55 casos (4). El objetivo del presente trabajo es comunicar los resultados de 500 labioplastías realizadas en el curso de más de 10 años de experiencia.

\section{PACIENTES Y MÉTODOS}

Se realizó un estudio de tipo descriptivo de los resultados obtenidos en el universo de labiobioplastías practicadas por dos cirujanos (JP y VS), con 386 y 114 intervenciones, respectivamente, entre octubre de 2003 y abril de 2014, en 5 clínicas de Santiago, Chile. En más de la mitad de los casos, la cirugía fue realizada por ambos médicos, pero habiendo adquirido mayor experiencia, se comenzaron a realizar por un solo cirujano y sin ayudante.

A pesar de que en una comunicación previa los autores establecieron una clasificación de hipertrofia de labios menores, en esta ocasión no se estimó necesario medir los labios para justificar la intervención, sino que se registraron los motivos por los cuales se decidió la cirugía. Éstos fueron catalogados como: razones estéticas, funcionales y psicológicas.

Las razones estéticas fueron definidas cuando no existían molestias locales ni de la esfera psicológica/sexual. Como causas funcionales se consideraron: incomodidad local inespecífica, roce inadecuado y molesto en actividades deportivas o con la ropa.

Los criterios de exclusión de la cirugía fueron: i. No haber alcanzado al menos dos años de pos menarquia, ii. Vulvitis aguda y iii. Tener un trastorno de autoimagen corporal. En cuanto a la selección para utilizar electrobisturí o láser, ésta tuvo relación a la disponibilidad del equipo láser y no a otro tipo de criterios.

Todas las pacientes recibieron información detallada acerca del procedimiento y posibles complicaciones, en la consulta previa a la cirugía, y firmaron un consentimiento informado específico.

Finalmente, para evaluar la satisfacción en cuanto a los resultados obtenidos, todas las pacientes fueron interrogadas dentro de los tres meses siguientes de la cirugía, donde se les solicitó responder acerca de su grado de satisfacción en relación a sus expectativas previas a la cirugía, de acuerdo a una escala ordinal que categoriza las opciones de respuesta en: Muy satisfecha, Satisfecha o Insatisfecha.

Técnica quirúrgica. La paciente se recuesta en posición ginecológica. De preferencia la paciente se recibe rasurada en la región genital para evitar la incomodidad que provoca el vello genital al realizar 
las suturas. Después de una aseptización vulvoperineovaginal el cirujano realiza una evaluación detallada de la anatomía de los labios con el objeto de tener claros los distintos pliegues, grosores y variaciones particulares. Estas son importantes, puesto que en la mayoría de los casos se requiere una resección de parte del prepucio del clítoris por los lados y, en ocasiones, al centro; lo que hemos denominado la "armonización del prepucio clitoridiano". En otros casos, son con frecuencia retirados los excesos de piel que se prolongan de los labios menores o que se presentan de manera independiente en la región que rodea el periné. Después de decidir las líneas de corte se realiza un dibujo marcando la zona interna y externa o posterior donde se van a realizar las incisiones (Figura 1). Enseguida, se confronta el lado interno del labio, marcado con el lápiz, con el contralateral para marcar la línea de corte. Esto tiene por objeto el buscar la mayor simetría posible, puesto que la simetría absoluta es muy difícil de lograr. La forma de realizar la incisión que se ha utilizado ha ido variando con el tiempo y experiencia adquirida. Durante los primeros 100 casos, los labios se extendían hacia los lados fijándolos con suturas a dos gasas que quedaban entre éstos y la cara interna del muslo. Posteriormente, se fue abandonando esta técnica por una más libre que varía de paciente en paciente. Con mayor frecuencia, actualmente se realiza el corte ayudando a la tracción con una pinza quirúrgica Hudson. En cuanto al instrumento de corte, corresponde a un láser de contacto (CLMD 60 Contact Laser Nd YAG laser and sapphire scalpels ER2; Surgical Laser Technologies Inc., Montgomeryville, PA, USA) con una potencia de corte promedio de $10 \mathrm{~W}$. Durante el año 2008, y con la experiencia de haber intervenido a aproximadamente 200 pacientes, se cambió el instrumento de corte por un electrobisturí con punta de tungsteno, Stryker (Colorado Needle $®$ ), con el cual se realiza el corte y coagulación, ambos con energías de 18 a $20 \mathrm{~W}$. Después de retirar el tejido sobrante se realiza una cuidadosa hemostasia del lecho coagulando con el electrobisturí. Posteriormente, se realiza el cierre de los labios con poliglactina 5-0 (en los primeros 250 casos se utilizó 4-0). La sutura de los labios también ha ido variando con el tiempo y no tenemos una norma, pues creemos que esta cirugía es diseñada a medida de cada paciente. El cierre se ha realizado con puntos separados, intradérmico o continuo externo. En los dos últimos casos, generalmente, se deben aplicar algunos puntos de refuerzo interrumpidos, con el mismo material. Otra variante que se aplica con frecuencia es lo que hemos denominado puntos de "memoria", término aprendido con el Dr. David Matlock del Laser Vaginal Rejuvenation Institute de Los Angeles, California y que consisten en dos o tres puntos de lado a lado en la base de los labios, que

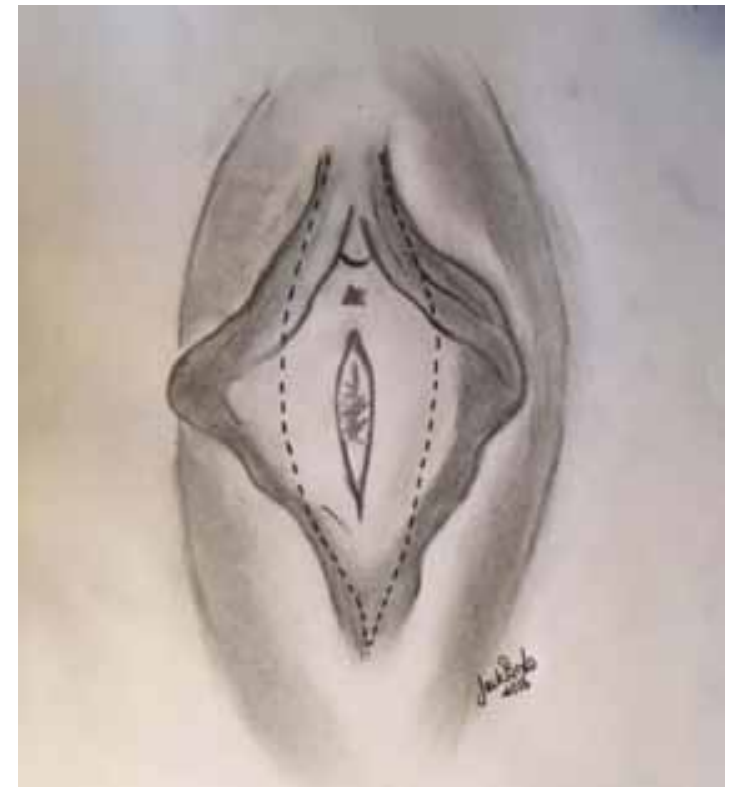

Figura 1. Líneas de corte iniciales, utilizadas en la mayoría de los casos de labioplastía.

tienen por objeto evitar la retracción y mantener el labio menor con el largo elegido.

Durante la operación se administra una dosis de Cefazolina. En el pos operatorio se administra ketoprofeno $100 \mathrm{mg}$ endovenoso y durante los primeros dos días se aplica hielo local, sin contacto directo con la piel y en forma intermitente. En las labioplastías únicas no se indican antibióticos profilácticos postoperatorios.

En cuanto a las indicaciones post cirugía, se autoriza a la paciente a ducharse a las 24 horas. Las medidas de aseo local indicadas enfatizan el uso de champú o jabón de ducha, que escurra por la zona, después el secado con toalla de algodón y luego secador de pelo. Se ha recomendado en todos los casos el uso de una crema cicatrizante que contiene centella asiática durante las primeras tres semanas. Se recomienda un reposo relativo en cama las primeras 48 horas y luego levantarse dentro de su casa. Por lo general, el retorno laboral se produce 4 a 5 días después de la operación, aunque en algunas pacientes en particular, es factible a las 48 horas.

La primera evaluación postoperatoria se realiza a la semana de la cirugía y luego al mes de operada, cuando por lo general, se da definitivamente de alta. Durante ese mes se indica evitar actividades deportivas que impliquen roce local, ropa apretada, relaciones sexuales y baños de inmersión de cualquier tipo, como tina, jacuzzi, piscina o mar. 


\section{RESULTADOS}

El rango de las pacientes fue de 10 a 72 años, $7 \%(\mathrm{n}=35)$ tenían menos de 20 años al momento de la cirugía. Una paciente de 10 años no llevaba 2 años de pos menarquía, pero el grado de hipertrofia era tan importante que se realizó la cirugía a solicitud de la madre.

Las indicaciones de labioplastía fueron estéticas en $95,4 \%(n=477)$, funcionales en $37,2 \%$ $(n=186)$ y psicológicas en $17,4 \%(n=87)$. Varios casos presentaban hipertrofia de labios mayor a 10 $\mathrm{cm}$, con casos de 14 a15 cm.

El $46,6 \%(n=233)$ de las labioplastías se asociaron a otra cirugía ginecológica, como: slings suburetrales, colpoperineoplastias por amplitud vaginal sintomática (rejuvenecimiento vaginal), prolapsos, histerectomías, miomectomías, esterilizaciones tubarias laparoscópicas y otras cirugías cosméticas (lifting de labios mayores y lipoaspiración de pubis). En $5,2 \%(n=26)$, hubo cirugías plásticas no ginecológicas asociadas como: abdominoplastías, lipoaspiraciones, mamoplastías e implantes mamarios, todas practicadas por cirujanos plásticos certificados no miembros del equipo ginecológico.

En 8 casos correspondieron a retoques o labioplastías de revisión, de los cuales 3 eran pacientes del equipo que no habían quedado totalmente satisfechas con la primera cirugía.

La anestesia administrada fue regional en el $88 \%(n=440)$, general en $10,4 \%(n=52)$ y local en $1,6 \%(n=8)$. Las anestesias generales se usaron preferentemente cuando hubo cirugías plásticas asociadas o histerectomías laparoscópicas.

El tiempo operatorio promedio fue de 40 minutos (rango: 24 a 67 minutos). No se presentaron complicaciones intraoperatorias en ningún caso. Asimismo, no hubo sangrado importante en ninguna labioplastía. En 2 casos hubo sangrado moderado postoperatorio no controlable por presión que hizo necesario aplicar un punto de sutura. En uno de los casos, el sangrado se presentó en la habitación antes del alta y el otro, al día siguiente en la consulta. En ambos casos se usó un anestésico local (lidocaína).

En todos los casos en que la labioplastía fue el único procedimiento, el alta se indicó en promedio 6 horas después de la cirugía. Cuando se combinó la labioplastía con otra cirugía, esta última fue la que comandó el alta médica.

En el mediano plazo, en $9 \%$ de los casos $(n=44)$ se produjo una dehiscencia leve a moderada de la sutura los que fueron manejados de manera conservadora, cerrando todas ellas espontáneamente.

Con estas medidas descritas el manejo del dolor fue muy exitoso. Sólo $2,8 \%$ de las pacientes $(n=14)$ refirieron dolor moderado por más de una semana, el que se resolvió espontáneamente. Sólo se registró un caso de dolor prolongado postoperatorio, el cual correspondió a una paciente con labios menores muy gruesos en que se hizo resección de tejido conectivo-celular con el objeto de adelgazarlos. El caso se resolvió aumentando los días de analgesia y uso de antiinflamatorio no esteroidal.

En cuanto al grado de satisfacción con el resultado obtenido a través de la cirugía, 96\% $(n=480)$ respondió que se encontraban muy satisfechas con los resultados de la cirugía, 3\% $(n=15)$ satisfechas y $1 \%(n=5)$ insatisfechas, aunque no arrepentidas de haberse realizado el procedimiento (Tabla I). A todas las pacientes que no quedaron totalmente satisfechas con el resultado estético, se les ofrece la realización de una corrección sin costo y hasta el momento sólo tres de ellas lo han solicitado y aceptado.

En relación a las molestias funcionales, todas las pacientes manifestaron sentirse muy cómodas con el resultado funcional y no relataron molestias posteriores.

Tabla I

RESUMEN DE DATOS EN 500 LABIOPLASTÍAS

\begin{tabular}{|c|c|c|c|}
\hline Variable & & & \\
\hline Edad & Promedio: 40 años & Rango 10-72 & $7 \%$ menores de 20 años \\
\hline Indicaciones & Estética 95,4 \% & Funcional $37,2 \%$ & Psicológica 17,4\% \\
\hline Asociación a otra cirugía & $53,6 \%$ no & $46,6 \% \mathrm{si}$ & - \\
\hline Instrumento de corte & Láser: $48 \%$ & Electrobisturí: $52 \%$ & - \\
\hline Complicaciones & Intraoperatorias : no & Sangrado postoperatorio $0,4 \%$ & - \\
\hline Retoque post-operatorio & No: $99,4 \%$ & Si: $0,6 \%$ & \\
\hline Anestesia & Regional: $88 \%$ & General: $10,4 \%$ & Local 1,6\% \\
\hline Tiempo operatorio & 40 minutos & Rango 24-67 minutos & \\
\hline Dehiscencia parcial & No: $91 \%$ & Si: $9 \%$ & \\
\hline Grado de satisfacción & Muy satisfechas: $96 \%$ & Satisfecha: $3 \%$ & Insatisfechas: $1 \%$ \\
\hline
\end{tabular}


En el $52,0 \%(n=260)$ de las intervenciones se realizaron utilizando electrobisturí y láser en el $48,0 \%(n=240)$, sin diferencias en cuanto a los resultados de satisfacción entre ambos procedimientos: en el grupo de pacientes que se mostraron "Insatisfechas" con la cirugía, en 3 se habían realizado utilizando laser y 2 con electrobisturí.

Hasta el momento no se ha registrado ninguna paciente que haya formado una cicatriz queloidea en sus labios y no han existido quejas referentes a disminución de la sensibilidad. Asimismo, no se ha recibido ningún caso de reclamo médico legal relacionado con esta cirugía.

En 10,4\% ( $n=52)$ de los casos se realizó estudio anatomopatológico de la muestra obtenida. En todos ellos el diagnóstico histológico mostró un cierto grado de componente inflamatorio, con variaciones en cuanto a su cronicidad u otros factores (Tabla II).
En Figuras 2, 3 y 4, se muestran los resultados estéticos antes (A) y después $(B)$ de tres casos sometidos a labioplastía vulvar.

\section{DISCUSIÓN}

El tema de las cirugías "cosméticas" ginecológicas ha estado de moda en los últimos años en los medios de prensa y comunicación. Si bien ha sido fuente de debate y controversia en la especialidad, la evidencia en los últimos años, apunta hacia los exitosos resultados que se obtienen en esta área. Así, un artículo publicado en la editorial de la Revista de la International Urogynecological Association (IUGA) en el año 2007, concluyó que la cosmetoginecología vino para quedarse y que el papel de las distintas sociedades y grupos serios de la especialidad ya no debía ser el oponerse, sino el de estudiarlo y estandarizarlo $(10,11)$.

Tabla II

HALLAZGOS ANÁTOMO-PATOLÓGICOS

\begin{tabular}{lc}
\hline Anatomía patológica & $\mathrm{n}$ \\
\hline Vulvitis crónica inespecífica & 30 \\
Vulvitis inespecífica bilateral & 13 \\
Vulvitis crónica inespecífica mas acrocordón de labio menor & 2 \\
Vulvitis crónica inespecífica y quistes epidérmicos vulvares & 1 \\
Vulvitis crónica con hiperplasia epidérmica & 1 \\
Vulvitis crónica con tejido de granulación focal sin atipias. & 1 \\
Vulvitis crónica con hiperplasia fibroepitelial & 1 \\
Vulvitis crónica asociada a hipertrofia de labios menores & 1 \\
Vulvitis crónica inespecifica con hiperplasia epitelial vulvar con leve incremento de la fibrosis estromal \\
asociada a congestión vascular \\
Dermatitis crónica superficial perivascular
\end{tabular}

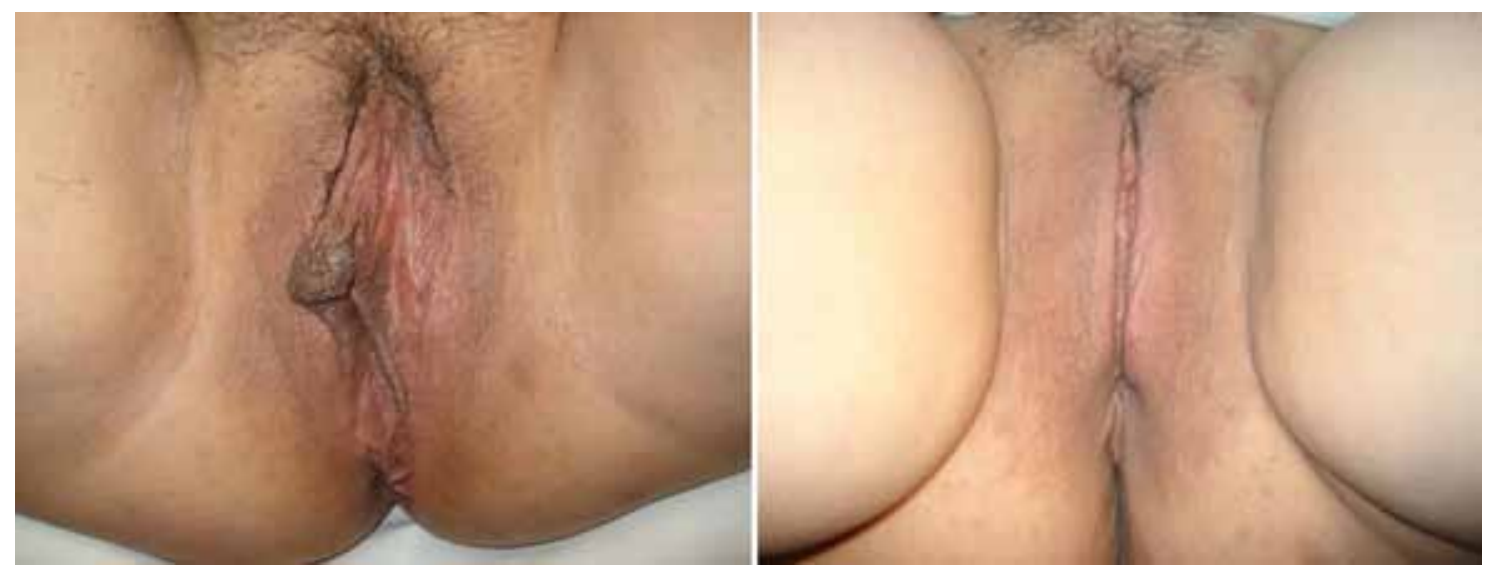

Figura 2. 61 años, labioplastia láser: A antes, B después. 

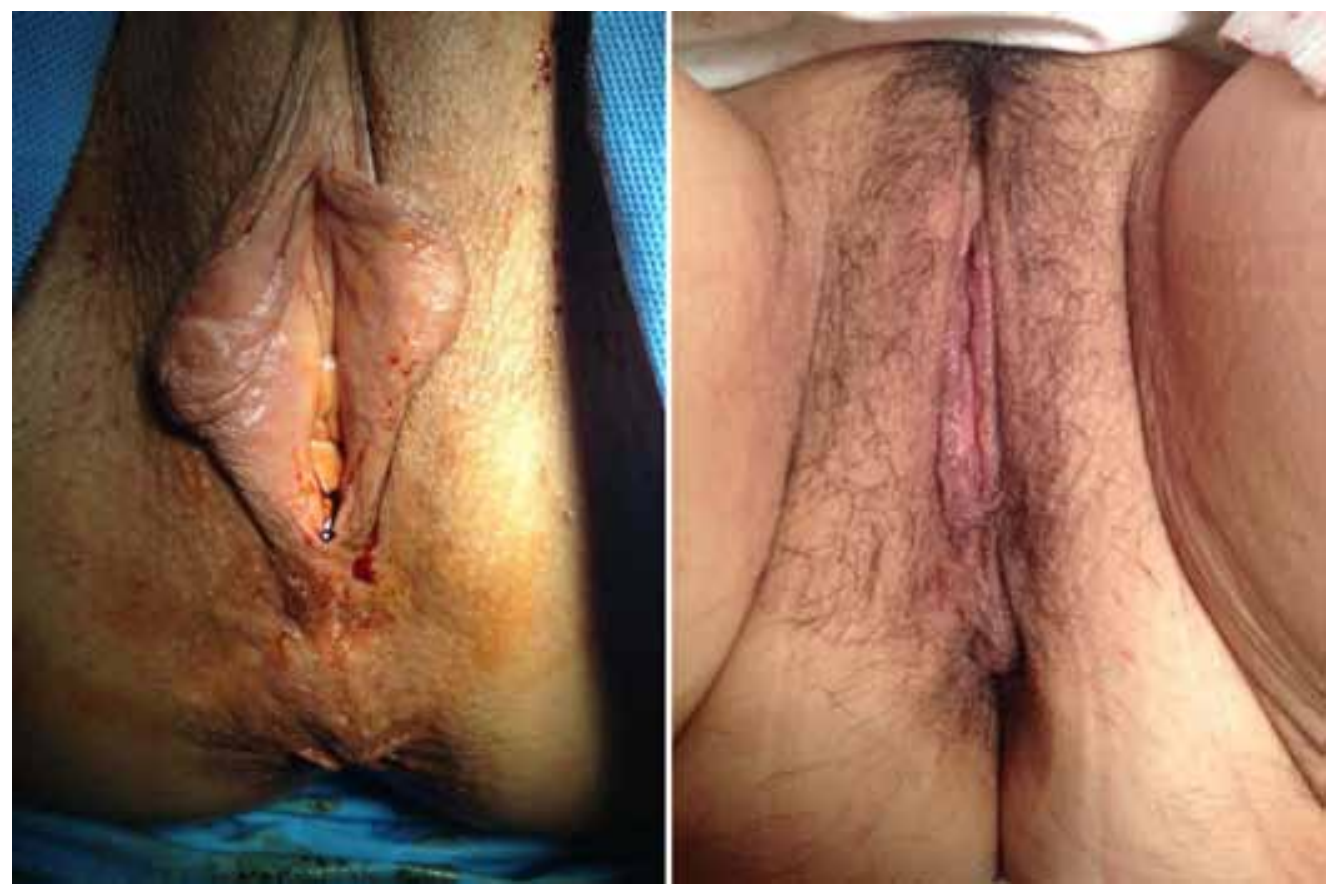

Figura 3. 25 años, labioplastia con electrobisturí: A antes y B después.

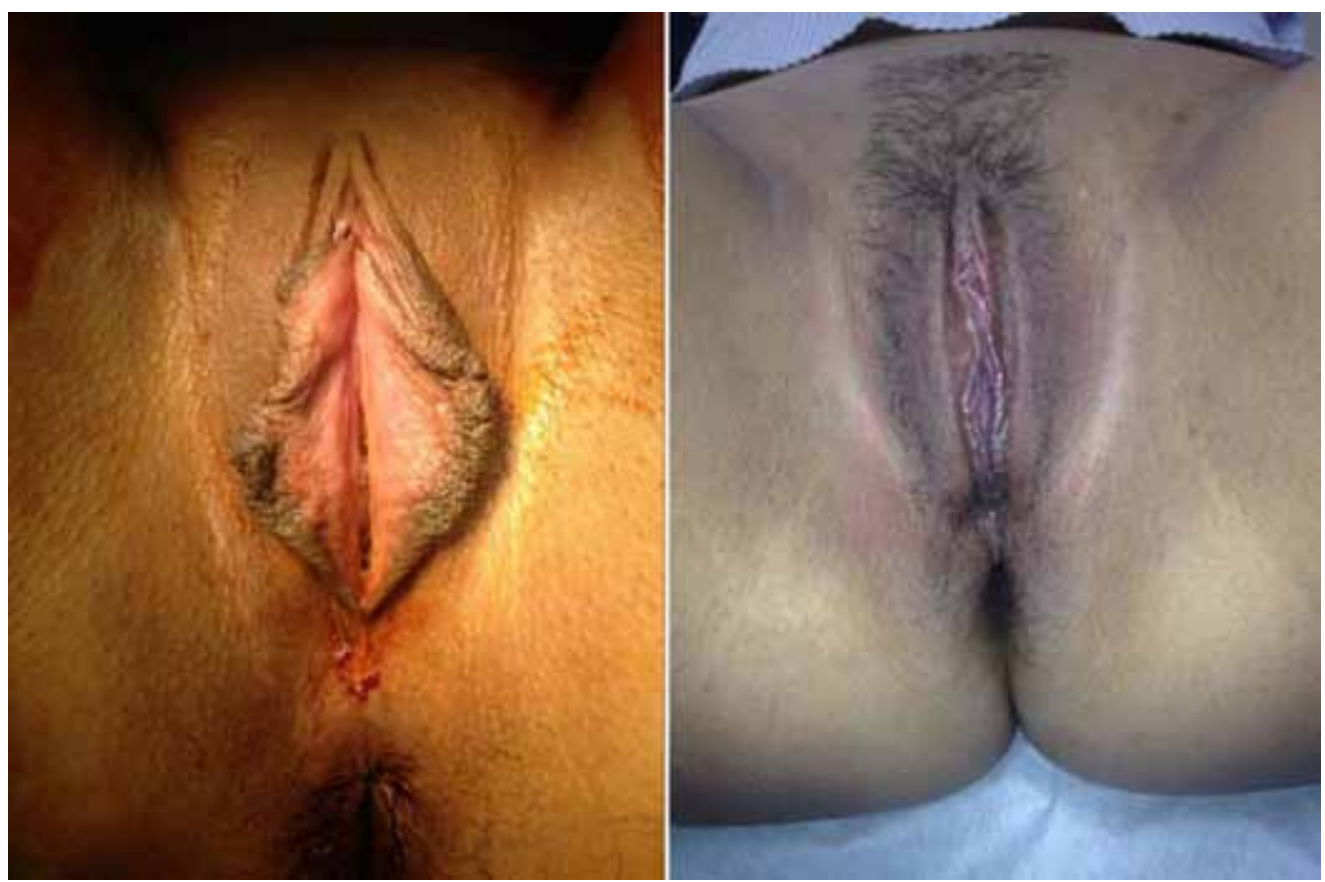

Figura 4. 38 años, labioplastia láser: A antes y B después. 
El presente estudio muestra los resultados obtenidos en la mayor serie de labioplastías con láser reportadas a la fecha en la literatura, nacional e internacional. Estos resultados muestran la alta satisfacción obtenida en cuanto a la resolución de la hipertofia de labios menores y sus implicancias en ámbitos estéticos, funcionales y psicológicos, cumpliendo ampliamente con las expectativas de las pacientes, lo cual es concordante con otros resultados publicados previamente sobre esta técnica. Recientemente, Miklos y cols (12), en un estudio prospectivo, mostraron que la labioplastía bien indicada proporciona excelentes resultados, con alto grado de mejoría en la autoestima y disminución del disconfort local, sin afectar negativamente el orgasmo.

En cuanto a la técnica quirúrgica presentada, a nuestro parecer, el diseño de las líneas de corte es el punto cardinal. Uno de los principales cuidados que tenemos en la cirugía es evitar la sobre corrección. Por otra parte, nos parece relevante explicar por qué no realizamos la técnica en " $Z$ ". Esto se debe a que en la mayoría de los casos la zona interna remanente de los labios menores presenta un color más claro. Las pacientes buscan tener los labios más claros posibles, rosados y con la técnica en $\mathrm{Z}$ el borde por lo general sigue siendo oscuro.

En cuanto a la utlización de láser versus electrobisturí, no hemos encontrado hasta ahora ventajas categóricas con alguno de éstos procedimientos, en cuanto a complicaciones, grados de satisfacción y resultado final estético.

Finalmente, queremos destacar que, la asociación a otras cirugías ginecológicas o cosméticas, es una práctica habitual no sólo en esta experiencia, sino también en otra serie reportada por los autores (13).

Pensamos que, así como ocurrió cuando hace 30 años las mujeres buscaron aumentar su volumen mamario con implantes y al poco tiempo esta cirugía, inicialmente rechazada, terminó siendo aceptada, enseñada y regulada, lo mismo debería ocurrir con la labioplastía. Los cirujanos de la especialidad no deberíamos objetar la reducción de labios menores de una mujer, psicológicamente sana, que siente un menoscabo en su autoestima, autoimagen corporal o molestias funcionales por el tamaño de sus labios, sean éstos los menores o mayores (14). Creemos que, como ginecólogos, tenemos, la obligación de empatizar con las molestias de estas pacientes y, si no estamos en condiciones de ofrecer una cirugía basada en experiencia, debemos derivarla a un profesional especializado en el tema y que cuente con la expertiz quirúrgica suficiente.

\section{CONCLUSIÓN}

A nuestro parecer, la hipertrofia de labios menores, aún cuando pueda considerarse subjetiva, es una condición que merece ser corregida cuando es requerido por la paciente y que las técnicas actuales ofrecen resultados seguros y satisfactorios.

\section{REFERENCIAS}

1. Iglesia CB, Yurteri-Kaplan L, Alinsod R, Female genital cosmetic surgery: a review of techniques and outcomes. Int Urogynecol J 2013;24:1997-2009.

2. Goodman M. Female genital cosmetic and plastic surgery: a review. J Sex Med 2011;8:1813-25.

3. Moore R, Miklos J. Vaginal rejuvenation and cosmetic vaginal surgery. En: Cardozo and Staskin (eds). Textbook of Female Urology and Urogynecology. $3^{\mathrm{a}}$ ed. London: Informa Healthcare, 2010; pp 1056-74.

4. Pardo J, Sola P, Guiloff E. Laser labiaplasty of the labia minora. Int J Gynecol Obstet 2005;93:38-43.

5. Davison SP, West JE, Caputy G, Talavera F, Stadelmann WK, Slenkovich NG. Labiaplasty and labia minora reduction. eMedicine.com 2008. Disponible en: http://reference.medscape.com/article/1372175overview Acceso: 24 de julio de 2015.

6. Giraldo F, Gonzalez C, De Haro F. Central wedge nymphectomy with a 90-degree Z-plasty for aesthetic reduction of the labia minora. Plast Reconstr Surg 2004;113:1820-7.

7. Chavis WM, La Ferla JJ, Niccolini R. Plastic repair of elongated, hypertrophic minora: a case report. J Reprod Med 1989;34:373-5.

8. Choil HY, Kim KT. A new method for aesthetic reduction of labia minora (the deepithelialized reduction labioplasty). Plast Reconstr Surg 2000;105:419- 22.

9. Miklos JR, Moore RD. Labiaplasty of the labia minora: Patient's indications for pursuing surgery. J Sex Med 2008;5:1492-5.

10. Pauls RN. Nip, tuck and rejuvenate: the latest frontier for the gynecologic surgeon. Int Urogynecol J 2007;18:841-2.

11. Pardo J, Solá V, Ricci P. Comments on Rachel N Paulus: "Nip, tuck and rejuvenate: the latest frontier for the gynecologic surgeon". Int Urogynecol J Pelvic Floor Dysfunct 2008;19:889-90.

12. Miklos J, Moore R, Chinthakanan O. Overall patient satisfaction scores, including sexual function, following labiaplasty surgery: A propspective study comparing women with a history of prior cosmetic surgery to those with none. Plastic \& Reconstructive Surgery 2014;134(4S-1):124-5.

13. Pardo J, Solá V, Ricci P. Cosmetics gynecological surgeries associated to mid-suburethral slings. J MinimaIly Invasive Gynecology 2008;15(6 Supplement):140S.

14. Pardo J, Solá V, Ricci P. Labia majora lifting: aesthetic and functional solution for hypertrophy and/ or flaccidity of labia majora. Am J Cosmetic Surgery 2012;29(2):128-32. 